\title{
TRATAMIENTO DE LA TUBERCULOSIS EN PEDIATRÍA. ACTUALIZACIÓN DE LA NORMATIVA CHILENA
}

Tuberculosis treatment in pediatrics. Update. Chile.

\section{Dra. Tania Herrera}

Directora del Programa de Tuberculosis

Ministerio de Salud

Chile

TUBERCULOSIS TREATMENT IN PEDIATRICS. UPDATE. CHILE.

We present a summary of Tuberculosis Prevention and Control Program's guidelines, which were updated in 2014, and are related to tuberculosis infection management in children under 15 years old, and their treatment in the case of active tuberculosis.

Key words: tuberculosis, children, therapeutics, chemoprophylaxis.

RESUMEN

Este es un resumen de las normas técnicas del Programa de Control y Eliminación de la Tuberculosis actualizadas el año 2014 y que tienen relación con el manejo de la infección de la tuberculosis en menores de 15 años y su tratamiento cuando se trata de una tuberculosis activa.

Palabras clave: tuberculosis, niños, tratamiento, quimioprofilaxis.

\section{INTRODUCCIÓN}

La tuberculosis en Chile se encuentra en una etapa particular, en la que a pesar de tener una incidencia baja en comparación con el resto de la región, no se ha podido superar la tasa de eliminación avanzada - 10 casos por 100.000 habitantes - que correspondía a la meta del año 2010. La tasa de incidencia de tuberculosis en todas sus formas se ha mantenido sobre 12 casos nuevos por 100.000 habitantes desde hace varios años, con estancamientos de la incidencia aún más importantes en los grupos etarios más jóvenes (1).

El grupo de menores de 15 años es considerado de manejo pediátrico en el Programa de Tuberculosis, estipulándose actividades y procedimientos distintos a los realizados en los adultos. Este grupo ha presentado tasas de incidencia de tuberculosis bajas, menor a 2 casos por 100.000 , con un número total de casos que se ha mantenido en 50 al año, con excepción del 2013 en que la cifra aumentó a 57 (1). En cuanto a la distribución geográfica, los casos infantiles no se presentan en todas las regiones, pero esta distribución es variable dependiendo, sobretodo, de los esfuerzos que se hagan en el estudio de contactos, que es la principal fuente de casos de tuberculosis infantil (2).

\section{Correspondencia:}

Dra. Tania Herrera

Ministerio de Salud

Maclver 541 - Santiago de Chile

Correo electrónico: tania.herrera@minsal.cl
Otro tema importante en este grupo de edad es el tratamiento de la infección tuberculosa 0 tuberculosis latente, tomando en cuenta que la primoinfección tiene altas posibilidades de avanzar hacia formas activas de la enfermedad, en especial en los menores de 5 años, y que la quimioprofilaxis puede evitarlo en hasta un $90 \%(3,4)$.

El presente artículo expone los lineamientos del Programa de Tuberculosis en relación al tratamiento de la enfermedad activa y latente en los menores de 15 años, destacando los principales cambios que se han realizado a la normativa en su actualización del año 2014. Su propósito es que el manejo de esta patología sea conocida por los especialistas en neumología infantil, más allá de los referentes del Programa de Tuberculosis, y además aportar a dar relevancia a un tema que ha permanecido en el olvido para muchos pediatras, pero que sigue presente en Chile.

\section{ESTUDIO DE CONTACTOS MENORES DE 15 AÑOS}

La tuberculosis infantil está siempre en relación a un adulto bacilífero que constituye la fuente de contagio, a lo que se agrega que el complejo primario suele ser asintomático, por lo que el estudio de contactos es fundamental para realizar el diagnóstico (2). El estudio de contactos es una actividad sistemática y normada por el Programa de Tuberculosis y es realizada en forma gratuita para todas las personas que habitan el territorio nacional, independiente de su condición previsional 0 migratoria y que cumplen con los criterios para definirse 
como un contacto de un caso índice de tuberculosis pulmonar o laríngea con confirmación bacteriológica (baciloscopía, cultivo 0 pruebas moleculares positivas). Cuando el caso índice es un menor de 15 años se realizará el estudio de contactos para todas las formas de tuberculosis (pulmonares y extra pulmonares) para buscar la fuente de contagio del menor.

Deben estudiarse todos los menores de 15 años que viven con el caso índice (contactos intradomiciliarios) y aquellos que por razones laborales, escolares o sociales mantengan relaciones frecuentes con el caso índice (contactos extradomiciliarios). El estudio se realiza al momento del diagnóstico del caso índice mediante una radiografía de tórax, una prueba de tuberculina (PPD) y la baciloscopía y cultivo en caso de que el contacto sea sintomático respiratorio. Los menores deben ser evaluados con estos exámenes por el neumólogo infantil de referencia del programa, quien están identificados en cada Servicio de Salud, y quien será el/la que determine si se trata de un caso de tuberculosis activo o si existe una infección tuberculosa sin enfermedad activa.

Una baciloscopía o cultivo positivo o una radiografía de tórax alterada en la que se observe un complejo primario u otras lesiones sugerentes de tuberculosis indican que se trata de un caso secundario y es necesario tratar. En caso de que la radiografía sea normal y la bacteriología negativa se procederá a efectuar quimioprofilaxis como se detallará más adelante. Cuando existen radiografías alteradas pero persiste alguna duda diagnóstica es posible solicitar baciloscopía de contenido gástrico como apoyo al diagnóstico.

El estudio de contactos de los casos multidrogorresistentes debe repetirse cada seis meses por 5 veces - al diagnóstico del caso y a los 6, 12,18 y 24 meses realizando radiografía de tórax y repitiendo el PPD si este fue no reactivo en el estudio anterior.

En el caso que se trate de recién nacidos hijos de madres con tuberculosis pulmonar, no debe separarse al niño 0 niña de su madre pero se le indicará a ésta el uso de mascarilla. Si la madre tiene baciloscopías negativas en al momento del parto se debe vacunar al recién nacido con BCG como lo indica el calendario de vacunación nacional. Si las baciloscopías de la madre son positivas y el recién nacido está clínica 0 radiológicamente sano, se realizará la quimioprofilaxis, posterior a lo cual se procederá con la vacunación.

\section{TRATAMIENTO DE LA TUBERCULOSIS ACTIVA EN MENORES DE 15 AÑOS}

El tratamiento de la tuberculosis en Chile se caracteriza por ser un tratamiento asociado, prolongado, directamente supervisado y gratuito para todas las personas que viven en el territorio nacional. El tratamiento de los casos menores de 15 años será indicado por el neumólogo infantil de referencia del programa, quien además debe controlar mensualmente al paciente, y será administrado bajo supervisión directa en el establecimiento de salud más cercano al domicilio del menor.
Los esquemas de tratamiento constan de dos fases: una fase diaria en que los fármacos se administran de lunes a viernes por 50 dosis, seguida de una fase trisemanal (lunes, miércoles y viernes) consistente en 48 dosis.

Los fármacos utilizados y los esquemas de tratamiento son similares a los de adultos, con la diferencia que las dosis por kilo de peso es mayor en los niños y pueden realizarse esquemas con menor número de fármacos dependiendo de la magnitud del compromiso pulmonar. Así, los fármacos utilizados corresponden a la rifampicina $(\mathrm{R})$, isoniacida $(\mathrm{H})$, pirazinamida $(\mathrm{Z})$ y etambutol (E). Las dosis por kilo de peso se muestran en la Tabla 1.

\begin{tabular}{|c|c|c|}
\hline FÁRMACO & FASE DIARIA & FASE TRISEMANAL \\
\hline Isoniacida & $10 \mathrm{mg} / \mathrm{Kg}$ & $15 \mathrm{mg} / \mathrm{Kg}$ \\
\hline Rifampicina & $15 \mathrm{mg} / \mathrm{Kg}$ & $20 \mathrm{mg} / \mathrm{Kg}$ \\
\hline Pirazinamida & $35 \mathrm{mg} / \mathrm{Kg}$ & \\
\hline Etambutol & $20 \mathrm{mg} / \mathrm{Kg}$ & \\
\hline
\end{tabular}

Tabla 1. Dosis de medicamentos utilizados para el tratamiento de la tuberculosis infantil.

La forma más benigna de enfermedad corresponde al complejo primario simple, el cual se tratará con el esquema primario simplificado: $2 \mathrm{HR} / 4 \mathrm{H} 3 \mathrm{R} 3$; en las tuberculosis no confirmadas bacteriológicamente se utilizará el esquema primario, pero con solo tres fármacos en la fase diaria: 2HRZ/4H3R3; en las tuberculosis más avanzadas, confirmadas por bacteriología, se utilizará el mismo esquema primario del adulto, ajustando las dosis por kilo de peso: 2HRZE/4H3R3.

\section{QUIMIOPROFILAXIS EN MENORES DE 15 AÑOS}

Toda indicación de quimioprofilaxis en menores de 15 años debe ser realizada por el neumólogo infantil referente del programa. Una vez realizado el estudio de contactos y habiendo descartado una tuberculosis activa, todos los menores de 15 años deben quedar con la indicación de quimioprofilaxis, la cual se realiza con isoniacida a una dosis diaria (lunes a domingo) de $5 \mathrm{mg} / \mathrm{Kg}$ (dosis máxima $300 \mathrm{mg}$ ) y en forma autoadministrada por 6 meses.

Aquellos niños que en el estudio de contactos inicial hayan presentado un PPD no reactivo (menor a $10 \mathrm{~mm}$ ), serán sometidos a un nuevo PPD a los 3 meses. Si el PPD continúa siendo no reactivo se suspenderá la quimioprofilaxis, en cambio 
si es reactivo deberá completarse la quimioprofilaxis por los 6 meses.

La isoniacida para la quimioprofilaxis será entregada una vez a la semana a un adulto responsable de adminístrasela al menor en el establecimiento de salud más cercano a su domicilio.

Para profundizar la información se recomienda consultar la norma técnica del Programa de Tuberculosis disponible en http://web.minsal.cl/tuberculosis

\section{El autor declara no tener conflicto de intereses}

\section{REFERENCIAS}

1. Programa Nacional de Control y Eliminación de la Tuberculosis. Informe de situación 2013. Ministerio de Salud. Chile

2. Cruz I, Velásquez J. Tuberculosis infantil. ¿Cómo diagnosticarla? Archivos argentinos de pediatría. 2012; 110:144-151

3. Sánchez G, Mamani R, Retamal J, Rojo A, Casar C. Formas clínicas de la tuberculosis infantil: Hospital Roberto del Río. 1989-2005. Rev Chil Enf Resp 2008;24:101-105

4. Rodríguez J. Tuberculosis latente. Rev Chil Enf Resp 2012; 28: $61-68$ 